\title{
Síndrome febril prolongado: un desafío para el infectólogo pediatra
}

Carmen Sandoval, Constanza Pinochet, Anamaría Peña, Marcela Rabello, Alejandra Prado y Tamara Viviani

\section{Fever of unknown origin: a challenge for the pediatric infectious diseases specialist}

Introduction: Prolonged febrile syndrome (PFS) is defined as fever 7-10 days, with initial study does not allow etiologic diagnosis. Objective: To describe the main causes of the PFS and its temporal behavior in Pediatric Infectious Diseases Unit Outpatient Care of Complejo Asistencial Dr. Sótero del Río (CASR). Patients and Methods: A descriptive, prospective study between january 2007-december 2012, about 153 patients from 6 weeks to 14 years 11 months old, diagnosed with PFS, tab completing clinical and laboratory monitoring. Results: etiology was obtained in $67.9 \%$, the causes were infection (88.4\%), neoplasms (4.8\%), rheumatological (4.8\%) and Kawasaki disease $(2.8 \%)$. The most important infectious causes were enteric fevers (typhoid and paratyphoid) (18.4\%), urinary tract infection (11.9\%), Bartonella henselae infections and adenovirus (8.7\%) each one and Epstein Barr virus (7.6\%). Ninety eight percent of patients had complete resolution, $60.7 \%$ did not require hospitalization and mortality was $0 \%$. Discussion: As in previous pediatric clinical series the infections were the most frequent causes. Enteric fever persists as principal cause, however, the epidemiological evidence is oscillating in time endorsing the local statistics can count over the years to improve the diagnostic and therapeutic approach.

Key words: prolonged febrile syndrome, fever of unknown origin, typhoid fever.

Palabras clave: síndrome febril prolongado, fiebre de origen desconocido, fiebre tifoidea.

\section{Introducción}

L a definición clásica de síndrome febril prolongado (SFP) es la presencia de fiebre, controlada, de al menos tres semanas de duración, y una semana de estudio exhaustivo, sin llegar a diagnóstico etiológico ${ }^{1,2,3}$. Esta definición es la más utilizada en la evaluación de adultos; sin embargo, en pediatría los meta-análisis y las revisiones más recientes se refieren a SFP o fiebre de origen desconocido (FOD) como aquel episodio febril de al menos 7-10 días de duración en el cual a pesar del estudio inicial, incluidos anamnesis, examen físico completo y exámenes de laboratorio básicos, no se ha llegado a un diagnóstico definitivo ${ }^{4,5}$.

Es importante, al momento de decidir estudiar a un paciente con SFP, aclarar y certificar esta condición ya que, frecuentemente ocurre que el paciente presenta cuadros febriles consecutivos, autolimitados, benignos, intercurrentes, en un período de tiempo que pueden aparentar un síndrome febril continuo y que, en la realidad, no cumple los criterios de fiebre prolongada ${ }^{4}$.

Tanto el estudio como el manejo y diagnóstico etiológico del SFP representan un desafío médico considerando la falta de consenso en las definiciones de esta entidad, la variabilidad etiológica según el sitio geográfico y los escasos reportes en la literatura médica ${ }^{4,6}$.
Respecto a la etiología, a pesar de lo comentado anteriormente, la causa más frecuente de fiebre prolongada es la infecciosa, seguida de patologías reumato-inmunológicas, neoplásicas y otras. En todas las publicaciones hay un grupo de pacientes que concluye su enfermedad sin diagnóstico etiológico a pesar de haberse efectuado un acabado estudio ${ }^{5,7)}$; habitualmente corresponden a cuadros prolongados, autolimitados y en general de buen pronóstico, especialmente si clínicamente hay mejoría y los exámenes de laboratorio van claramente a la normalización ${ }^{4}$. Es interesante destacar que siendo la causa infecciosa la más frecuente, ésta se constituye en fiebre prolongada de difícil diagnóstico, no por corresponder a un cuadro originado por un agente poco frecuente, sino más bien, porque siendo un agente común, tiene una presentación inhabitual ${ }^{1,8}$.

En algunos reportes se ha realizado el análisis de los pacientes con SFP por grupos de edad, destacando que éste sería un factor importante al momento de la orientación diagnóstica y es así como se publica que, en pacientes bajo dos años de edad, la causa más frecuente de SFP sería la infecciosa y a mayor edad tendrían más relevancia otras etiologías 5 .

En el año 2007, nuestro grupo de trabajo, publicó un estudio de $\mathrm{SFP}^{9}$, que analizó pacientes del mismo grupo etario y zona geográfica que el actual, pero con otro
Complejo Asistencial Dr. Sótero del Río, Santiago, Chile. Sub-dirección Médica del Niño Unidad Ambulatoria de Infectología (CSC, MR).

Pontificia Universidad Católica de Chile, Santiago, Chile. Facultad de Medicina División de Pediatría (CP, AP, AP, TV).

Los autores declaran no tener conflictos de intereses. Estudio sin financiamiento.

Recibido: 5 de julio de 2013 Aceptado: 10 de diciembre de 2013

Correspondencia a: Tamara Viviani Salgado tviviani@med.puc.cl 
panorama epidemiológico debido a la diferente temporalidad. En dicho estudio la causa más frecuente fue la infecciosa, destacando Bartonella henselae, infección del tracto urinario (ITU) y virus de Epstein Barr (VEB), llamando la atención la ausencia de infecciones sistémicas frecuentes en Chile como fiebre tifoidea y enfermedades neoplásicas, hallazgo explicado probablemente por el momento epidemiológico que abarcó el estudio y por el número de pacientes incluídos.

El presente reporte es un estudio prospectivo en población infantil que cumplió criterios de SFP, atendidos en la unidad ambulatoria de infectología de la Sub-dirección Médica del Niño (SDMN) del Complejo Asistencial Dr. Sótero del Río (CASR), en un período de tiempo de seis años.

El objetivo de la presente publicación fue describir las principales etiologías del SFP y su comportamiento temporal, en este centro asistencial, con el propósito de ordenar el estudio y manejo de este síndrome de acuerdo a nuestra realidad.

\section{Pacientes y Métodos}

Estudio descriptivo, prospectivo, de pacientes con edades entre 6 semanas y 14 años 11 meses, evaluados en la unidad ambulatoria de infectología de la SDMN del CASR entre enero de 2007 y diciembre de 2012, con diagnóstico de SFP. Se definió SFP como: fiebre $\geq 7$ días en niños entre 6 semanas y 4 años $\mathrm{y} \geq 10$ días en niños $>$ 4 años, sin diagnóstico clínico ni de laboratorio previo al momento de su inclusión. Se excluyeron pacientes con el antecedente de: inmunodeficiencias, neoplasias, patología reumatológica e historia de síndromes febriles periódicos. Según fuesen la evolución clínica y la sospecha diagnóstica, se realizaron estudios y controles seriados hasta llegar a un diagnóstico definitivo y/o la resolución total de la fiebre, normalización de los exámenes y mejoría del paciente.

A todos los pacientes se les completó una ficha de seguimiento que incluyó: antecedentes médicos, examen físico y exámenes de laboratorio iniciales (hemograma, VHS, proteína $\mathrm{C}$ reactiva (PCR), perfil bioquímico, radiografía de tórax, hemocultivo, sedimento de orina y urocultivo), evolución clínica y exámenes de seguimiento según correspondiese (detección de virus respiratorios, coprocultivo, mielograma, mielocultivo, serologías [Mycoplasma pneumoniae, B. henselae, VEB, citomegalovirus (CMV), parvovirus B19, Toxoplasma gondii, test de Widal-reacción de aglutinación, y otras], baciloscopias, PPD, estudio de imágenes (ecotomografía abdominal, ecografía cardíaca, cintigrama óseo, TAC de cavidades paranasales u otros, radiografía de huesos largos y calota).

\section{Definiciones}

- Enfermedad por arañazo de gato: manifestaciones clínicas compatibles e IgG (+) para $B$. henselae $\geq 1: 256$ (mediante técnica de IFI, valor positivo $\geq 1: 256$; Laboratorio Pontificia Universidad Católica de Chile).

- Enfermedad por Mycoplasma pneumoniae: tos y sintomatología respiratoria persistente por más de una semana, con estudio de virus respiratorios negativo (panel respiratorio mediante IFI; Laboratorio Hospital Sótero del Río) y serología IgM (+) para M. pneumoniae (mediante técnica de ELISA, valor positivo $>1,1$; Laboratorio Pontificia Universidad Católica de Chile).

- Enfermedad por VEB: poliadenopatías, con faringoamigdalitis exudativa y serología IgM $(+)$ cualitativa para VEB (mediante técnica de ELISA, valor positivo $>$ 1,1; Laboratorio Pontificia Universidad Católica de Chile).

- Fiebre tifoidea: hemocultivo y/o mielocultivo y/o coprocultivo positivo(s) para Salmonella Typhi ó Paratyphi y/o test de Widal con títulos superiores a: anti-O $\geq 1: 160-200$ y/o anti-H $\geq 1: 160-200^{10}$.

- Enfermedad por CMV: serología IgM (+) para CMV (mediante técnica de ELISA, valor positivo $>1,1$; Laboratorio Hospital Sótero del Río).

- Toxoplasmosis: serología IgM (+) para T. gondii (mediante técnica de ELISA, valor positivo $>1,1$; Laboratorio Hospital Sótero del Río).

- Parvovirus: serología IgM (+) para parvovirus B19 (mediante técnica de IFI, valor positivo $\geq 1: 16$; Laboratorio Pontificia Universidad Católica de Chile).

\section{Resultados}

En el período de estudio se incluyeron en el protocolo 153 pacientes, con un promedio de edad de 4,4 $\pm 3,9$ años. Del total, 80/153 (52,3\%) correspondieron al género masculino. Los días de fiebre al momento de ingresar al protocolo fueron en promedio 13,5 \pm 8 días. La duración total de la fiebre fue en promedio 17,6 \pm 11 días. La Tabla 1 muestra la descripción general de la población de estudio.

Se obtuvo diagnóstico etiológico en 67,9\% (104/153) de los pacientes (Figura 1). Las causas más frecuentes fueron infecciones $(92 / 104$ casos; $88,4 \%)$, seguidas de neoplasias y reumatológicas $(4,8 \%$ cada una) y finalmente la enfermedad de Kawasaki (2,8\%). Cabe destacar que un paciente presentó dos diagnósticos, uno oncológico y otro infeccioso en forma simultánea (cáncer de colon y enfermedad por VEB).

Entre los 92 pacientes con una causa infecciosa identificada, las fiebres entéricas (tifoidea y paratifoidea) fueron las más frecuentes $(17 / 92 ; 18,4 \%)$, seguida de ITU $(11,9 \%)$, infecciones por $B$. henselae y adenovirus (ADV) en igual proporción $(8,7 \%)$, enfermedad por $\operatorname{VEB}(7,6 \%)$, luego enfermedades causadas por M. pneumoniae, CMV, 
gingivo-estomatitis herpética y sinusitis ( $5,4 \%$ cada una), neumonía y síndrome diarreico $(4,3 \%$ cada una), infección por virus parainfluenza $(3,2 \%)$ y otras infecciones (síndrome disentérico, síndrome mononucleósico (SMN) clínico, bacteriemia por Leuconostoc sp, bacteriemia por Staphylococcus aureus, otitis media aguda, síndrome mano-pie-boca, adenitis por $S$. aureus y adenitis mesentérica (en total 10,9\%).

La mayoría de las fiebres entéricas fueron diagnosticadas en épocas de primavera-verano y los pacientes tenían una edad promedio de 7,1 años; consultaron con un promedio de 12,3 días de fiebre (rango: 7-22) y 3/17 de ellos correspondieron a infección por $S$. Paratyphi; los pacientes restantes correspondieron a $S$. Typhi. El cuadro clínico fue, en general, típico. Siete de los 17 pacientes tuvieron hemocultivos positivos, 4 de ellos para $S$. Typhi y 3 para $S$. Paratyphi; un paciente tuvo coprocultivo positivo para $S$. Typhi, con hemocultivo negativo. En los restantes 9 pacientes, los títulos de antígeno anti-H o anti-O fueron ${ }^{3} 1: 160$, destacando que en 7 de ellos los títulos alcanzaron a ${ }^{3} 1: 320$. No se practicó mielocultivo ni se realizó curva serológica.

Entre los pacientes con enfermedades por $B$. henselae, la edad promedio fue de 7,7 años y consultaron con un promedio de 19,7 días de fiebre (rango: 8-60), 2/3 refirieron contacto con gatos, tres de ellos se presentaron como enfermedad por arañazo de gato diseminada (uno con espondilodiscitis y dos con granulomas esplénicos).

Los niños con enfermedad por ADV tuvieron una edad promedio de 1,7 años y consultaron con un promedio de 10,5 días de fiebre (rango: 8-21). En todos, la manifestación clínica fue una faringo-amigdalitis asociada a adenopatías cervicales; ninguno presentó un SMN clásico.

Los pacientes con enfermedad por VEB tuvieron una edad promedio de 3,9 años y consultaron con un promedio de 13,5 días de fiebre (rango: 10-20). Todos desarrollaron un SMN clásico.

Los casos con CMV tuvieron una edad promedio de 2,7 años y consultaron con un promedio de 16 días de fiebre (rango: 8-23). En ellos sólo destacó la fiebre como principal síntoma y no presentaron un SMN clásico.

Por último, en los niños con infección por M. pneumoniae la edad promedio fue 5,8 años y consultaron con un promedio de 12,6 días de fiebre (rango: 7-22). La presentación clínica fue extremadamente variable, predominando la tos como síntoma principal.

La Tabla 2 destaca las principales etiologías infecciosas y su comparación con la serie clínica antes publicada por Peredo y cols ${ }^{9}$. En esta Tabla se aprecia, como principal diferencia entre ambos períodos, el resurgimiento de la fiebre tifoidea como una causa importante de fiebre prolongada.

La Figura 2 muestra la variabilidad año a año de las principales etiologías infecciosas como causa de SFP, destacando el dinamismo de este síndrome.
Tabla 1. Descripción general de la población de niños con síndrome febril prolongado. Complejo Asistencial Sótero del Río, años 2007-2012
Número de pacientes enrolados

Edad (años)

Género

Masculino

Femenino

Duración de la fiebre al momento del enrolamiento (días)

Duración total de la fiebre (días)

\section{3}

Promedio 4,4 \pm 3,9

Mediana 2,6 años (3 meses-14 años)

$\begin{array}{lc}n & \% \\ 80 & 52,3 \\ 73 & 47,7\end{array}$

Promedio $13,5 \pm 8$

Mediana $11(7-60)$

Promedio 17,6 \pm 11 Mediana 14 (8-67)

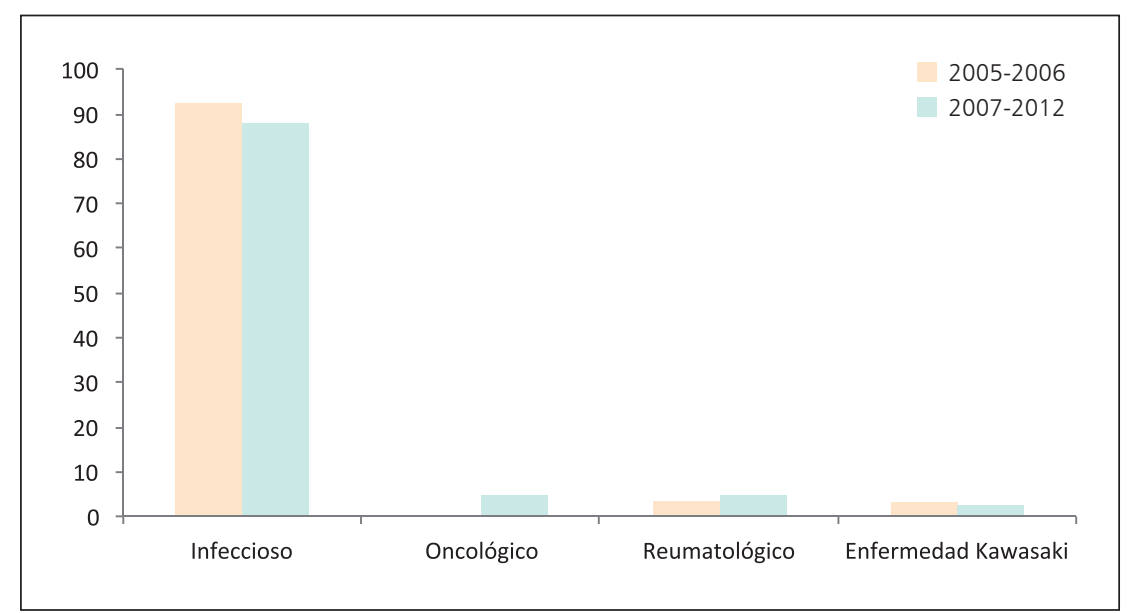

Figura 1. Comparación de las etiologías infecciosas, oncológicas y reumatológicas del síndrome febril prolongado en niños (6 semanas-14 años 11 meses) en dos períodos de estudio (2005-2006 y 2007-2012). Complejo Asistencial Sótero del Río.

Tabla 2. Principales etiologías infecciosas del síndrome febril prolongado en niños en estudio 2005-2006 y estudio 2007-2012, Complejo Asistencial Sótero del Río

\begin{tabular}{|c|c|c|}
\hline Etiologías infecciosas & $\begin{array}{l}\text { Peredo y cols. } \\
(2005-2006)\end{array}$ & $\begin{array}{c}\text { Sandoval y cols. } \\
(2007-2012)\end{array}$ \\
\hline Fiebre tifoidea & $1 \quad(4,1 \%)$ & $17(18,4 \%)$ \\
\hline Infección por Bartonella henselae & $6(25 \%)$ & $8 \quad(8,7 \%)$ \\
\hline Infección por adenovirus & 0 & $8 \quad(8,7 \%)$ \\
\hline Infección del tracto urinario & $4 \quad(16,6 \%)$ & $11(11,9 \%)$ \\
\hline Infección por virus de Epstein Barr & $2 \quad(8,3 \%)$ & $7 \quad(7,6 \%)$ \\
\hline Infección por citomegalovirus & 0 & $5 \quad(5,4 \%)$ \\
\hline Infección por Mycoplasma pneumoniae & $1 \quad(4,1 \%)$ & $5 \quad(5,4 \%)$ \\
\hline Gingivo-estomatitis herpética & 0 & $5 \quad(5,4 \%)$ \\
\hline Sinusitis aguda & $1 \quad(4,1 \%)$ & $5 \quad(5,4 \%)$ \\
\hline Otras & $9 \quad(37,8 \%)$ & $21 \quad(23 \%)$ \\
\hline Total & $24(100 \%)$ & $92(100 \%)$ \\
\hline
\end{tabular}




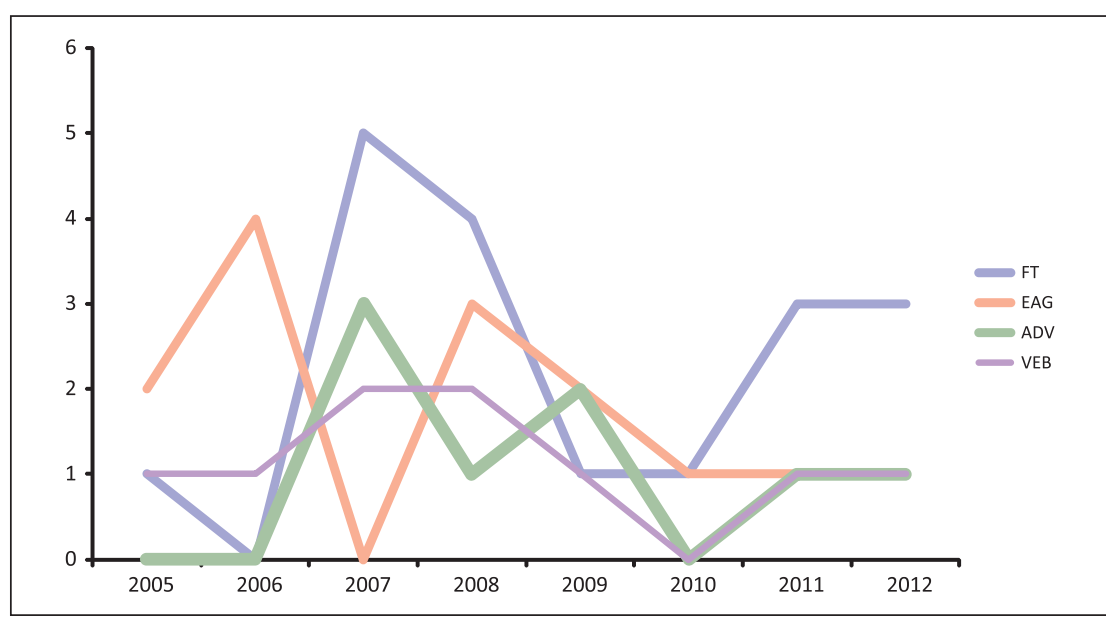

Figura 2. Comportamiento temporal de las principales causas infecciosas de síndrome febril prolongado en niños. Complejo Asistencial Sótero del Río.

Los pacientes con artritis reumatoídea tuvieron una edad promedio de 10 años y se presentaron con un promedio de 10 días de fiebre (rango: 8-19). Un paciente cursó con efusión pericárdica y pleural; en el resto de los pacientes predominó la fiebre como principal sintomatología.

Los niños con diagnósticos onco-hematológicos tuvieron una edad promedio de 7,9 años y se presentaron en promedio con 20 días de fiebre (rango: 8-30); todos se presentaron con adenopatías asociados a compromiso del estado general y sudoración nocturna. Los diagnósticos fueron leucemia linfoblástica aguda, linfoma y cáncer de colon.

En 49 pacientes (32\%) no fue posible determinar una etiología; sin embargo, en $98 \%$ de ellos hubo una resolución completa del cuadro. En tres pacientes no hubo posibilidad de certificar la resolución clínica del SFP ya que no acudieron a control; no obstante, se aseguró en todos, por búsqueda de estadística del centro, que no tuviesen nuevas consultas ni hospitalizaciones por SFP al menos en los seis meses siguientes, del ingreso al presente protocolo. El 60,7\% de los pacientes fue evaluado sólo en forma ambulatoria, seriada y sistemática. No hubo fallecidos mientras se llegó al diagnóstico causal del SFP o hasta la resolución de la fiebre.

\section{Discusión}

En esta serie se corrobora que la etiología infecciosa es la causa más frecuente de SFP en pediatría, correspondiendo a más de la mitad de los casos y esto se mantuvo durante un período de ocho años en niños atendidos en un hospital público del área suroriente de Santiago. Chow y cols., en un meta-análisis publicado el 2011 que incluyó 1.638 niños, confirmó que la etiología infecciosa (51\%) era la causa que más comúnmente explicaba el $\mathrm{SFP}^{5}$. Generalmente corresponde a infecciones comunes cuyo diagnóstico se retrasa por varios motivos, entre otros, un estudio incompleto, su expresión clínica inhabitual o la falta de sospecha clínica por inexperiencia en el tema, del médico tratante.

En la casuística de este estudio, la causa infecciosa más frecuente fue la infección sistémica por Salmonella spp, seguida de ITU, bartonelosis y enfermedades causadas por $A D V$ y VEB. Cabe destacar que la fiebre entérica, a pesar de haberse presentado en bajo porcentaje en el estudio publicado el año 2007, vuelve a adquirir relevancia en éste, delatando el patrón característico de esta enfermedad, es decir, su presentación oscilante a través del tiempo ${ }^{8,9,11}$. No hubo diferencias del porcentaje de patología infecciosa entre los niños bajo dos años versus el grupo etáreo $\geq$ dos años, a diferencia de otras series 5 .

Las etiologías neoplásicas y reumatológicas se presentaron en la misma proporción, situación variable en los distintos reportes, incluidos los nuestros, probablemente relacionado con el número de pacientes analizados. Aunque constituyan una causa poco frecuente de fiebre prolongada, es importante recalcar que deben ser siempre considerados en el estudio del paciente con SFP por su implicancia clínica.

Los resultados de este estudio dan cuenta de una realidad algo distinta a reportes de países en vías de desarrollo, como India, donde muchas de las patologías que se presentan como SFP presentan gran morbilidad, e incluso mortalidad, que puede fluctuar entre 3 y $4 \%$; en general, en países con bajos niveles de desarrollo la mortalidad por cuadros infecciosos comunes y prevenibles aún tiene un peso importante. En nuestra serie, la ausencia de mortalidad puede explicarse, en parte, por el grado de desarrollo económico y social de nuestro país, que ha llevado a políticas sanitarias de alta calidad y con gran impacto en la morbi-mortalidad de patologías infecciosas comunes prevenibles.

En nuestro centro fue posible el seguimiento de la mayoría de los pacientes (dos tercios) en forma ambulatoria, realizando un control clínico y de exámenes seriados y reevaluación permanente, tomando la decisión de hospitalización cuando correspondía; esto permitió evitar el riesgo de complicaciones asociadas a la atención de salud por la internación.

En todo paciente con SFP es fundamental el seguimiento, ya que, en un porcentaje importante no es posible llegar a un diagnóstico específico. En este grupo en particular, debe observarse tanto la evolución clínica como de los exámenes de laboratorio hasta su normalización y tenerse presente que, si bien la mayoría de ellos tendrá una evolución favorable, uno de cada 10 pacientes puede presentar un diagnóstico final con pronóstico reservado (reumatológico y/o neoplásico). Todos los pacientes de 
este estudio que quedaron sin diagnóstico etiológico (32\%), resolvieron el cuadro febril y ninguno cursó con deterioro clínico ni de laboratorio. A todos estos pacientes (excepto en tres de ellos) se les realizó seguimiento adecuado verificando la resolución completa del cuadro.

La comparación de las dos series estudiadas por nuestro equipo, efectuadas sobre una misma población, pero en años distintos (2005-2006 la primera y 2007-2012 la segunda), refleja la variabilidad epidemiológica en el tiempo y en un mismo lugar de las patologías, resaltando la importancia de realizar estudios permanentes que faciliten la mejor orientación diagnóstica y el enfrentamiento del pediatra a este tipo de cuadros ${ }^{12}$. Los pacientes consultantes en nuestra institución representan una condición socio-económica en que co-existen las etiologías infecciosas clásicas propias de países en desarrollo y patologías propias de países desarrollados. Esta situación toma mayor importancia debido a que, en estudios japoneses la etiología infecciosa no es la más frecuente sino que predomina la etiología reumatológica, poniendo mucho más en énfasis el deber realizar estudios locales ${ }^{12}$.

Finalmente, pero no menos importante, es tener experiencia en este tema, lo que permite establecer una buena relación médico-paciente, de confianza, en el contexto que el SFP ocasiona incertidumbre tanto en el personal médico como en los padres ${ }^{13}$, tomando en cuenta además, que el paciente probablemente será sometido a varios estudios antes de tener un diagnóstico, mientras los padres observan que su hijo continúa enfermo, con fiebre, sin diagnóstico y sin tratamiento. Lo anterior nos lleva a destacar lo importante que es tener el concepto de que el SFP, tal como su nombre lo indica, es un síndrome clínico con variadas formas de presentación y múltiples etiologías, y que si bien la mayoría puede ser de causa infecciosa con evolución favorable, en un porcentaje el pronóstico pudiese ser ominoso, lo que exige de un compromiso del médico tratante con el paciente y su familia hasta tener un diagnóstico final y su tratamiento o la resolución completa del cuadro.

Agradecimiento. A todos los Residentes de Pediatría y de Infectología Pediátrica, por su ayuda permanente en llenar la ficha de seguimiento clínico de cada uno de los pacientes incluídos en el protocolo.

\section{Resumen}

Introducción: El síndrome febril prolongado (SFP) se define como fiebre entre 7-10 días, con estudio inicial que no permite un diagnóstico etiológico. Objetivo: Describir las principales etiologías del SFP y su comportamiento temporal en la unidad de infectología pediátrica ambulatoria del Complejo Asistencial Dr. Sótero del Río (CASR). Pacientes y Método: Estudio descriptivo, prospectivo, entre enero de 2007-diciembre de 2012. Análisis de 153 pacientes entre 6 semanas y 14 años 11 meses de edad, con diagnóstico de SFP, que completaron ficha de seguimiento clínico-laboratorial. Resultados: Se obtuvo diagnóstico etiológico en $67,9 \%$, las causas fueron: infecciones $(88,4 \%)$, neoplasias $(4,8 \%)$, reumatológicas $(4,8 \%)$ y enfermedad de Kawasaki $(2,8 \%)$. Las causas infecciosas más importantes fueron: fiebres entéricas (tifoidea y paratifoidea) $(18,4 \%)$, infección del tracto urinario (11,9\%), enfermedades por Bartonella henselae y adenovirus $(8,7 \%)$ cada uno y virus de Epstein Barr (7,6\%). El 98\% de los pacientes tuvo resolución completa, $60,7 \%$ no requirió hospitalización y no se registraron decesos. Discusión: Como en las series clínicas antes publicadas, las infecciones fueron la causa más frecuente de SFP. La fiebre entérica persiste como causa principal; sin embargo, se evidencia una situación epidemiológica oscilante en el tiempo justificando la necesidad de contar con estadísticas locales a lo largo de los años para mejorar el enfoque diagnóstico y terapéutico.

\section{Referencias bibliográficas}

1.- Long S S. Distinguishing among prolonged, recurrent and periodic fever syndromes Approach of a Pediatric Infectious Diseases subspecialist. Pediatr Clin North Am 2005; 52 (3): 811-35.

2.- Antoon J W, Bradford K K. Fever of unknown origin in a child. Clin Pediatr (Phila) 2013; 52 (1): 99-102.

3.- Seashore C J, Lohr J A. Fever of unknown origin in children. Pediatr Ann 2011; 40 (1): 26-30.

4.- Tolan R W Jr. Fever of unknown origin: a diagnostic approach to this vexing problem. Clin Pediatr (Phila) 2010; 49 (3): 207-13.
5.- Chow A, Robinson J L. Fever of unknown origin in children: a systematic review. World $\mathrm{J}$ Pediatr, 2011; 7 (1): 5-10.

6.- Joshi N, Rajeshwari K, Dubey AP, Singh T, Kaur R. Clinical spectrum of fever of unknown origin among Indian children. Ann Trop Paediatr 2008; 28 (4): 261-6.

7.- Antoon J W, Knudson-Johnson M, Lister W M. Diagnostic approach to fever of unknown origin. Clin Pediatr (Phila) 2012; 51 (11): 1091-4.

8.- Moya F, Talesnik E, Larco R. Fiebre prolongada en preescolares y escolares. Rev Chil Pediatr 1980; 51 (3): 174-8.

9.- Peredo G M S, Viviani S T, Peña D A. Etiología del síndrome febril prolongado en niños. Rev Chil Pediatr 2007: 78 (5): 472-6.
10.- Katime Zúñiga A E. Reacción de Widalinterpretación clínica. Rev Panam Infectol 2006; 8 (2): 40-4.

11.- Ruiz Goikoetxea M, Urabayen R, Herranz M, Moreno L, Gil FJ, Bernaola E. Imported infectious diseases in fever of unknown origin in migrant children: 2 cases. An Sist Sanit Navar 2012; 35 (1): 149-53.

12.- Kasai K, Mori M, Hara R, Miyamae T, Imagawa T, Yokota S. National survey of childhood febrile illness cases with fever of unknown origin in Japan. Pediatr Int 2011; 53 (4): 421-5.

13.- Cogulu O, Koturoglu G, Kurugol Z, Ozkinay F, Vardar F, Ozkinay C. Evaluation of 80 children with prolonged fever. Pediatr Int 2003; 45 (5): 564-9. 